\title{
Information systems requirements specification through the mediation of technical and organizational perspectives
}

\author{
António Lucas Soares \\ INESC/Manufacturing Systems Engineering Unit and \\ FEUP/DEEC - University of Porto, R. José Falcão, 110, 4050 \\ Porto - Portugal. Tel: +3512 2094300; Fax: +35122008487 \\ Email:asoares@inescn.pt,Web:www.inescn.pt/ asoares
}

\begin{abstract}
This paper introduces a conceptual modelling approach for supporting the requirements analysis and specification of information systems according to technical and socio-organisational perspectives, particularly in manufacturing enterprises. The approach is based in the so-called Techno-Organisational Development Ontologies that act as a conceptual core for perspectives mediation. A case study based on semi-conductor virtual enterprises is presented illustrating the validity of the approach.
\end{abstract}

\section{Keywords}

Information systems development, conceptual modelling, social and organizational aspects, ontologies

\section{INTRODUCTION}

Interdisciplinary teams should drive comprehensive technical and organisational change, and enterprise's workers should participate actively and thoroughly in 
this process. This requires methods and tools that support effectively interdisciplinarity and participation, and that help to manage the inherent complexity of such comprehensive approaches. We call Techno-Organisational (TO) development to the process of developing simultaneously technology, organisation and people in an enterprise, towards improved efficiency and effectiveness in pursuing the enterprise's mission (examples of TO development range from Business Process Reengineering to Continuous Process Improvement). Ideally, these processes should involve the application of engineering and social sciences in a "symbiotic" way (Benders et al., 1995). Enterprise Modelling, for example, is an important instrument, but currently its major focus is on engineering with minor insights in organisational and people issues. The work presented in this paper proposes that a shared understanding of the technoorganisational domain is the key to collaboration and participation support. This calls for conceptual modelling methods capable of taking into account the different social and organisational perspectives, together with the technical ones. Nevertheless, it would be unfeasible to conceptualise all the representative perspectives off the enterprise's socio-organisational intervention, as incompleteness and inadequacy would necessarily emerge. The approach shortly presented here emphasises the construction of a conceptual core providing a basic shared understanding and from which the several perspectives in action in a given TO development process would be specialised. We begin by describing the notion of perspectives mediation and its implementation through Ontological Engineering and afterwards by describing a case study concerning the development of a planning and control system for semi-conductor virtual enterprises.

\section{MEDIATION OF PERSPECTIVES IN INFORMATION SYSTEMS REQUIREMENTS SPECIFICATION}

Requirements analysis and specification for IS in e.g., manufacturing enterprises, always call for some modelling method(s) application in order to provide a structure and a shared understanding of the domain(s) being analysed. For example, when considering Enterprise Modelling (EM) as a means to support the requirements analysis and specification phases, it is generally agreed that current mainstream EM deals only with the technical part of the business processes. Nevertheless, it is very difficult in these phases to clearly cut between "technical part" and "organizational part", as the requirements for technical systems involve organisational requirements and vice-versa. One major research topic in technoorganisational development is the collaboration between development disciplines through methodologies and IT tools. One way to address this topic is through the development of comprehensive methodologies, encompassing the technical, social and organisational aspects, on what can be called methodological integration. This approach can lead to reductionism as it is not possible to completely and consistently consider all the development perspectives regarding technical and 
organisational analysis. One alternative is to consider methodological mediation instead of integration, and to develop frameworks for perspectives understanding and communication. Such a kind of approach is developed in the following sections.

\subsection{Ontologies as a framework for perspectives mediation}

The multitude of technical, human, social and organisational contexts that can be found in Enterprises, constrain the TO development courses in ways that frequently hinder the use of comprehensive methodologies. It is thus necessary to overcome the problems raised by specific technical languages used in representing and communicating domain conceptualisations, method outcomes, etc. One possible role of ontologies (in the sense of knowledge representation and sharing) is the one of communication medium between different people working together for a given purpose. This is the case of a TO development where teams composed by end users and external developers have to collaborate throughout the development life cycle.

\section{A generic socio-organisational conceptualisation}

The broad range of the socio-organisational domain and the multitude of theoretical and practical views on it, originate difficulties in its conceptualisation, unless we admit an ontological commitment to a given view. The notion of conceptual adequacy - the balance between generality and specificity in domain conceptualisation - is of utmost importance here. If one pretends to provide a conceptual core that enables the representation of different perspectives, it should provide the adequate generality and, at the same time, to embody an ontological commitment that does not constrain the type of social and organizational aspects that can be representable. Giddens structuration theory (Giddens, 1993) can be the foundation for such socio-organisational conceptualisation. Through this theory, an analysis of the social action and its links to the social structure production and reproduction can be made. The particular interpretation of structuration theory presented here, is twofold: firstly, it appears as the base for the basic conceptualisation of the enterprise's social interaction and structure, and secondly as a means of explanation of some organisational change issues, including the ones influenced by Information Technology (DeSanctis and Poole, 1994). We cannot present here in detail our application of structuration to Enterprise socioorganisational modelling (see Soares, 1998) but we can summarise it in the following the basic points. Enterprises are systems of interaction - which are characterised by communication, power and morality - within a structure whose characteristics are signification, domination and legitimation. The central idea advanced by Giddens is the duality of structure: "[...] structure is both constituted by human agency and at the same time is the very medium of this constitution" (Giddens, 1993, pp. 129). The fundamental issue is not to describe an Enterprise's organisation as a static structure, but as a system of (social) interaction that has 
structural properties enabling and constraining that interaction. In the other side, the action and interaction of social actors within the enterprise continuously reconstitute its structure.

\section{Techno-Organisational Development Ontologies}

The socio-organisational conceptualisation was partially formalised in a socioorganisational ontology (SOO) that includes social structure, social actor, and social action and interaction as its main parts. Social structure central entities include rule, resource, domain-of-discourse, modality and qualification. In the social actor part, social actors (which can be individuals or groups) are characterised in detail focusing on its intentions, goals, and attitudes. Finally, social action and interaction part defines the entities social-relation, powerrelation and interaction-domain as the main ones. These entities enable the modelling of Enterprise's socio-organisational aspects going from the formal (bureaucratic) issues - organisational units structure, task allocation and responsibilities, etc. - to more "soft" (yet tangible) issues such as power relations and motivational characteristics of jobs. Also, the concept of modality and its subconcepts (such as norms, facilities and interpretative-schemes), mediating between interaction and structure, are useful for modelling important socioorganisational issues. Examples of such issues are the inter-relationships of information systems (software systems) features with the system of social interaction: information systems features can be characterised in terms of the resources that they provide to social-actors, the organisational norms that they enforce or exempt and the schemes of interpretation that they provide in communication (roughly, the interpretative scheme is the domain of discourse (ontology) of a given pattern of interaction e.g., quality, planning, etc.).

Together with the SOO, two further ontologies were developed as to be the basis of collaboration tools in TO development projects: the System Requirements Specification Ontology (SRSO) and the Methodology Ontology (MO) resulting in a library called Techno-Organisational Development Ontologies (TODO). Its purpose is to act as a "core" representation i.e., a basic shared understanding for all the actors in a TO development process. It intends to include a comprehensive set of concepts, relationships and axioms that are fundamental for representing, communicating and reasoning about the technical and socio-organisational aspects of an innovation process in a company (see Figure 1). A powerful feature of ontological engineering is reuse. This means that every ontology doesn't need to start from scratch, but it can include terms already worked in other ontologies. For this work, we included in TODO the Enterprise Ontology (Uschold, 1995), developed by the University of Edinburgh (AIAI) whose defined terms on activities, resources and processes were found particularly useful. 


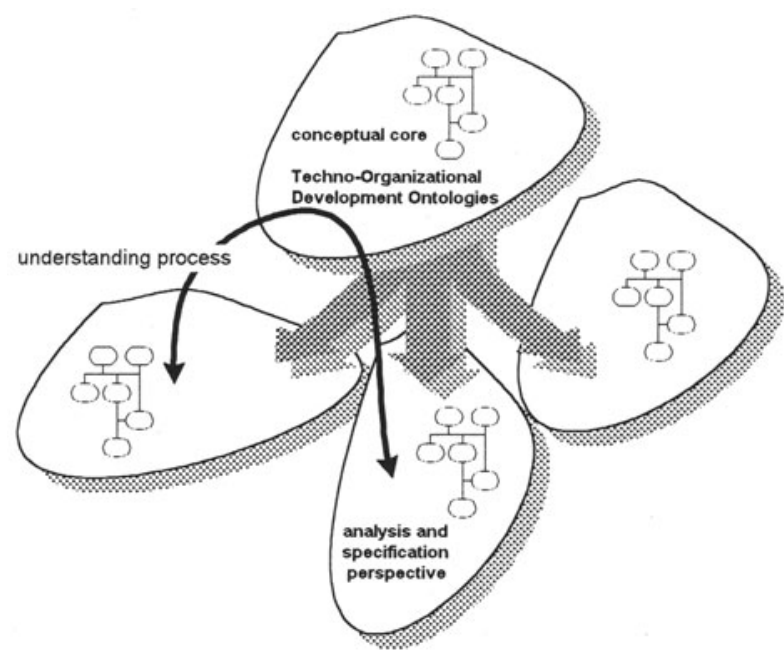

Figure 1 - Mediation of perspectives in techno-organisational analysis and specification.

The formalisation of the ontologies in a neutral knowledge representation language such as Ontolingua enables the automatic translation for a range of frame-based knowledge representation systems. The use of such tools provides the opportunity to develop collaborative artifacts featuring some form of automated reasoning (e.g., automated classification of concepts, complex queries, etc.) for helping in maintaining the consistency of models and in discovering new information through inference capabilities. We specified and built a prototype of such tool resulting in the caTOd (collaborative artifact for Techno-Organisational development) system. CaTOd is a knowledge based system based that uses the Techno-Organisational Development Ontology, to support the TO development by: helping the development actors to manage the development methods and models information, facilitating the ontologies' appropriation, facilitating the construction of domain models as specialisation of the ontologies, and answering to complex queries that can be made about the domains represented.

\section{A CASE STUDY}

An application of the ideas above explained is now presented through an illustrative case study. The case is drawn from a project funded by the European Commission under the ESPRIT programme (EP 205444 - X-CITTIC) that aimed at developing a planning and control system (PPC-VE) with special characteristics for use in semi-conductor (SC) manufacturing companies. These companies experienced global markets for a long time and have been establishing corporations based on networks of firms, or what can be called "Virtual Enterprises" (VE). Two companies were analysed. Firstly, a global analysis of the organisational change processes in the $\mathrm{SC}$ companies is made, resulting in the 
statement of some concerns related with the restructuring process, namely with the labour division, participation and qualification. Then, a qualitative analysis of the planning and control jobs characteristics highlights the individual motivational aspects to look at in the design of the new techno-organisational structure.

\subsection{Business Process Restructuring in SC Virtual Enterprises}

As a general trend in the SC industry, SC companies experience a major shift from a manufacturing driven business to a business driven one, with more than one business competing for manufacturing capacity. This change involves the total enterprise, although relying heavily on improved logistics, planning and control functions through restructuring and reorganisation. Concerning the enterprise's external relations, there is a trend to concentrate on fewer customers and have them as key customers in which on-going business is maintained through joint $\mathrm{R} \& \mathrm{D}$ or engineering application work. At the same time, the supplier base is also being drastically cut to secure better co-operative arrangements in terms of quantity, price and delivery. The major shift from manufacturing centric views to business centric views and activities is one of the motivations for the global restructuring and reorganisation. The restructuring processes rely heavily on an extensive rationalisation of the organisation's functional aspects, most of the times without explicit concerns in addressing the human and social aspects of change. Three basic questions can be raised.

\section{Labour division in the planning and control functions}

The future generic business process seems to be the best way to deal with the actual and future market conditions (unpredictability, quick response) and industrial structure (globalisation) within the SC business. Nevertheless, the division of tasks between a central planning and the manufacturing units in terms of planning and control seems a major factor in the success of this approach. A "total" centralising strategy, concentrating all the planning and control decision making can be dangerous in two ways. First, it can heavily constrain the overall agility of the company, in the sense that some decisions are shifted away from the place where the knowledge (not only formal, but also tacit) exists e.g., plan repair actions at the manufacturing units; this may lead to longer reaction times, moreover even inadequate actions. Second, the content of planning and control jobs at the manufacturing units is very much simplified, which could lead to a lower motivation potential of the planning and control tasks; besides lowering the quality of working life of the people assigned to these tasks, this can originate demotivation and de-responsibility within the organizational units with the inevitable consequent loss in both efficiency and effectiveness. 
Formalisation of the planning and control tasks

One of the expected BPR outcomes is an extensive formalisation for the planning and control tasks. Besides rationalisation issues, one major goal is preventing the problems arising from people unavailability. This can also lead to a certain loss in flexibility, if associated to a strict assignment of tasks. The later can also contribute to a decrease in the people's experienced responsibility of the work outcomes, with the consequent de-motivation.

\section{Strict top-down approach}

Organisational change is very difficult to result in outstanding improvements unless a strong people's commitment at all levels in the company is achieved. Participation is a crucial social process if the business relies on the people's competence and ingenious skills. Attempts to undertake extensive restructuring processes without such commitment is the reason under many BPR sub-optimal results or even failures. Technological innovation, if driven only by management decisions, can have as consequence a great deal of people's resistance when it comes to implement a new information system. This was the case of e.g., a well known PPC system introduction and actual implementation in one of the companies analysed in this case study which ran into difficulties due to a late information to the users, followed by poor training actions. Poor participatory culture can also lead to strategic moves from people involved in the change process e.g., the formation of IT legacy systems "interest groups" defending their knowledge and trying to extend their range of application in order to improve their power status.

\subsection{Social analysis of the planning and control related jobs}

The general concerns expressed above were a motivation for introducing a socioorganizational perspective in the PPC-VE requirements analysis and specification. With the intention of assessing the stakeholders perceived job motivation, a set of open interviews and daily work observation were undertaken. Although not following strictly any method, a very simplified approach based on the multiple paradigm organisational research proposed by Hassard (1993, pp. 88ff.) was used to get empirical data. This approach reflects the functionalist-interpretative paradigm opposition in sociology, being the functionalist "part" based upon the social systems theory and the interpretative one on ethnomethodology based approaches. In this particular case, we combined the job characteristics approach (Hackman and Oldman, 1980) with the observation and users explanation of their daily work. The data gathered was not sufficient for any rigorous assessment of the planning and control job characteristics but was significant enough to have an idea of their motivating potential. Nevertheless, this information helped to devise how the planning and control system could be designed to contribute to well design jobs in motivational terms. Table 1 summarises the conclusions of the interviews in the two companies. 


\begin{tabular}{|c|c|c|}
\hline core job characteristic & Users perceived level - $A$ & users perceived level $-B$ \\
\hline $\begin{array}{l}\text { skill variety - variety of } \\
\text { different activities } \\
\text { involves the use of } \\
\text { multiple skills and } \\
\text { talents }\end{array}$ & $\begin{array}{l}\text { Low - the variety of skills } \\
\text { to undertake the } \\
\text { planning tasks is not } \\
\text { so demanding. }\end{array}$ & $\begin{array}{l}\text { Moderate - some range of } \\
\text { skills is needed for } \\
\text { undertaking the } \\
\text { planning and control } \\
\text { tasks }\end{array}$ \\
\hline $\begin{array}{l}\text { task identity - degree to } \\
\text { which the job requires } \\
\text { the completion of a } \\
\text { "whole" and } \\
\text { identifiable piece of } \\
\text { work }\end{array}$ & $\begin{array}{l}\text { Low to moderate - the task } \\
\text { division at this } \\
\text { department causes } \\
\text { some fragmentation }\end{array}$ & $\begin{array}{l}\text { moderate to high - the } \\
\text { planning tasks form a } \\
\text { whole piece of work: } \\
\text { the plans are the final } \\
\text { result. }\end{array}$ \\
\hline $\begin{array}{l}\text { task significance - the } \\
\text { impact and importance } \\
\text { of the job within the } \\
\text { organisation }\end{array}$ & $\begin{array}{l}\text { Moderate to high - there is } \\
\text { a recognition of the } \\
\text { planning and control } \\
\text { tasks importance }\end{array}$ & $\begin{array}{l}\text { moderate to high - there is } \\
\text { a recognition of the } \\
\text { planning and control } \\
\text { tasks importance }\end{array}$ \\
\hline $\begin{array}{l}\text { autonomy - degree of } \\
\text { discretion to determine } \\
\text { how to perform the } \\
\text { assigned tasks }\end{array}$ & $\begin{array}{l}\text { Low to moderate - some } \\
\text { autonomy in the } \\
\text { procedures but a } \\
\text { concentration of } \\
\text { decision-making in } \\
\text { some persons }\end{array}$ & $\begin{array}{l}\text { moderate to high autonomy } \\
\text { in the decisions the } \\
\text { planners are } \\
\text { responsible for }\end{array}$ \\
\hline $\begin{array}{l}\text { feedback from job - } \\
\text { degree of direct and } \\
\text { clear information about } \\
\text { the effectiveness and } \\
\text { performance of the } \\
\text { tasks }\end{array}$ & $\begin{array}{l}\text { Low - not very much } \\
\text { information about the } \\
\text { quality of the } \\
\text { generated plans and } \\
\text { consequences of plan } \\
\text { repairing actions }\end{array}$ & $\begin{array}{l}\text { low to moderate - there is } \\
\text { some difficulty in } \\
\text { assessing the quality } \\
\text { of the generated plans } \\
\text { and consequences of } \\
\text { plan repairing actions }\end{array}$ \\
\hline
\end{tabular}

Table 1 - Users perceived level of the planning and control jobs internal characteristics.

\subsection{Modelling for multiple perspective analysis}

We exemplify now how the conceptual core provided by TODO can be used to span a shared conceptualisation of the social structure formed by the relevant social-actors in the planning, manufacturing and marketing organisational units and PPC-VE structural features.

The focus will be on the representation of the labour division (i.e., the planning and control work roles) and on its motivational characteristics as resulting from a job design survey method application. The resulting models convey important information for the specification of a system that improves the motivation potential of the planning and control work roles. The influence of PPC-VE structural features on the social structure of the planning and control related organizational units is also shortly analysed in the light of the TODO (terms in italic mean classes and relations defined in the ontologies). 


\section{Work organization and motivational analysis}

The first step is the discovery of the relevant social-actors. In this case, those are the persons (individuals) in the planning, manufacturing control and marketing/sales departments, the groups (formal or informal, temporary or permanent, intra or inter-departments) and the company itself. Representing the information about the work motivation evaluation method is achieved by specialising concepts and relations from MO such as core-job-characteristic $\subset$ method-result, analyse-interview $\subset$ development-activity or job-design-survey $\subset$ development-method. These are related to the work organization (work-roles, activities and its assignment to social-actors) by the following assertions: planning $\in$ work-role, skill-variety $\in$ core-job-characteristic, hascharacteristic(planner,skill-variety). This way we are able to represent, in the same conceptual model, a conventional activities' structure and assignment to organizational units (identified by some technical system analyst) and the social characterisation of work organization (undertook by some socio-organisational experts).

\section{PPC-VE structuration possibilities}

The information system will surely influence the domination, signification and legitimation properties of the planning, sales and manufacturing organizational units' social structure. Although this influence cannot be positively anticipated it is useful for the analysts to be able to explicitly represent the structuration possibilities for later discussion of the design options (DeSanctis and Poole, 1994). As explained above, the structuration possibilities of the planning and control system arise from the resources that social actors can found on it, the norms it enforces or exempts and the communicational context it provides. Concerning the "moral" aspects of interaction, one can foresee e.g., the system as providing norms on the prioritisation of demands or on stocking policies. This will involve directly the social-actors on the sales and planning departments but also indirectly the manufacturing departments. The interpretation of these norms by them will influence the legitimation aspect of the social structure. Another example is the influence of the information system on the signification properties of the social structure. The system provides an interpretative scheme (or several) in this case a planning scheme - that is likely to change the communication aspect of interaction. We can have an idea of the scheme provided by looking e.g., at the object model of PPC-VE, that represents roughly the planning ontology of the system. Less foreseeable from specification diagrams (such as OMT analysis models) would be the influence on the domination characteristics. Nevertheless, the resources provided by a comprehensive global planning system feature, will probably affect the power relations between the planning and manufacturing departments. The outcome of this analysis can be represented through the specialisation of the SOO and the SRS, in the way shortly illustrated in the previous section. 


\section{DISCUSSION}

The approach described in this paper tackles the social and organisational aspects representation in information systems analysis and specification from the theoretical grounds of the social theory. This enabled the creation of a socioorganisational ontology detached from any specific organisational perspective, but able to play the role of a conceptual core, spanning specialisations for any perspective. Together with a requirements and specification ontology, a methodology ontology and the enterprise ontology, forming what we called the Techno-Organisational Development Ontologies, a framework is set-up for creating tools which enable to place a bigger and more comprehensive effort in the analysis phases of system's development.

Techno-organisational approaches to information systems development are not "mainstream" yet, due to several factors from which the most important is the complexity of the process and the wide range of competencies needed both from the consultants and internal developers. Through the work shortly described here, we expect to contribute to an effective interdisciplinarity in the development through the support to the perspectives understanding and methodological mediation.

\section{REFERENCES}

Benders, J. et al. (1995) Symbiotic Approaches: Contents and Issues in The Symbiosis of Work and Technology, Benders et al. (Eds.), Taylor \& Francis.

De Sanctis, G., Poole, M. (1994) Capturing the Complexity in Advanced Technology Use: Adaptive Structuration Theory, in Organization Science, Vol. 5 2, pp. 121-147.

Giddens, A. (1993), New Rules of Sociologic Method, $2^{\text {nd }}$ Ed., Polity Press, UK. Hackman, J., Oldham, G. (1980) Work Redesign, Addison-Wesley.

Hassard, J. (1993) Sociology and Organization Theory: Positivism, Paradigms and Postmodernity, Cambridge University Press, London.

Soares, A. (1998) The role of structuration theory in supporting the analysis and design of advanced information systems for manufacturing. Proc. 6th Intl. Conf. on Human Aspects Of Advanced Manufacturing: Agility \& Hybrid Automation, Hong-Kong, China.

Uschold, M., King, M., Moralee, S., and Zorgios, Y. (1996) The Enterprise Ontology (Version 1.1). Technical Report, AIAI, University of Edinburgh.

\section{BIOGRAPHY}

António Soares received the $\mathrm{PhD}$ in Electrical and Computers Engineering in 1998 in the University of Porto. His main research interests are the social and organisational aspects of information systems development in manufacturing enterprises. 